\title{
Diseño y desarrollo de una aplicación como herramienta innovadora para el aprendizaje de la ley de los gases ideales
}

\section{Design and development of an application as an innovative tool for learning the ideal gas law}

Walther Geovanny Gamba-Cifuentes ${ }^{1}$; Luis Alejandro Quemba-Guavita²; Oscar Jardey Suárez³

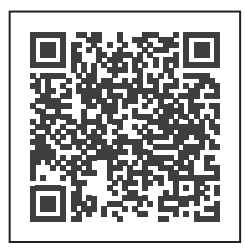

\section{Palabras clave:}

ambiente de la clase, educación básica, enseñanza de la física, recursos educativos abiertos, relación profesor-alumno, tecnología educacional.

Artículo de investigación científica y tecnológica.

Fecha de recepción: 06/10/2020

Fecha de aceptación: 23/02/2021

Esta publicación se encuentra bajo licencia:

Creative Commons Reconocimiento-

NoComercial-

SinObraDerivada 4.0 Internacional

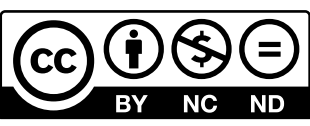

\section{Resumen}

El objetivo de este trabajo es reportar el diseño y desarrollo de una aplicación en el sistema iOS para el estudio de la ley de gases ideales con estudiantes de undécimo grado de una escuela privada en Bogotá, Colombia, debido a que, en los últimos años, dicha temática ha generado niveles bajos de aprendizaje en la población estudiantil. Por lo tanto, se diseñó una aplicación como herramienta didáctica, que pueda fortalecer el aprendizaje. Diferentes fuentes de trabajo señalan que el uso de las tecnologías de la información y la comunicación, como las aplicaciones, potencialmente puede favorecer el aprendizaje. La metodología seguida para el diseño y desarrollo es ISD-MeLO, que se enfoca en el esquema y progreso de contenidos educativos y se basa en el modelo de diseño instruccional ADDIE; las fases son: 1. Análisis; 2. Diseño; 3. Desarrollo; 4. Implementación; y 5. Evaluación. La aplicación fue

Licenciado en Física. Estudiante, maestría en Educación en Tecnología, Universidad Distrital Francisco José de Caldas. Docente de Ciencias Naturales, Colegio San Bartolomé la Merced, Colombia.wggambac@correo.udistrital.edu. CO ORCID: 0000-0002-9096-5169

2 Licenciado en Física. Estudiante, maestría en Educación en Tecnología, Universidad Distrital Francisco José de Caldas. Docente, de cátedra de Física, Pontificia Universidad Javeriana, Colombia. laquembag@correo.udistrital.edu. co ORCID: 0000-0001-9438-4612

3 Doctor en Educación con Énfasis en Ciencias. Docente, maestría en Educación en Tecnología, Universidad Distrital Francisco José de Caldas, Colombia. ojardeys@correo.udistrital.edu.co ORCID: 0000-0001-8780-595X 


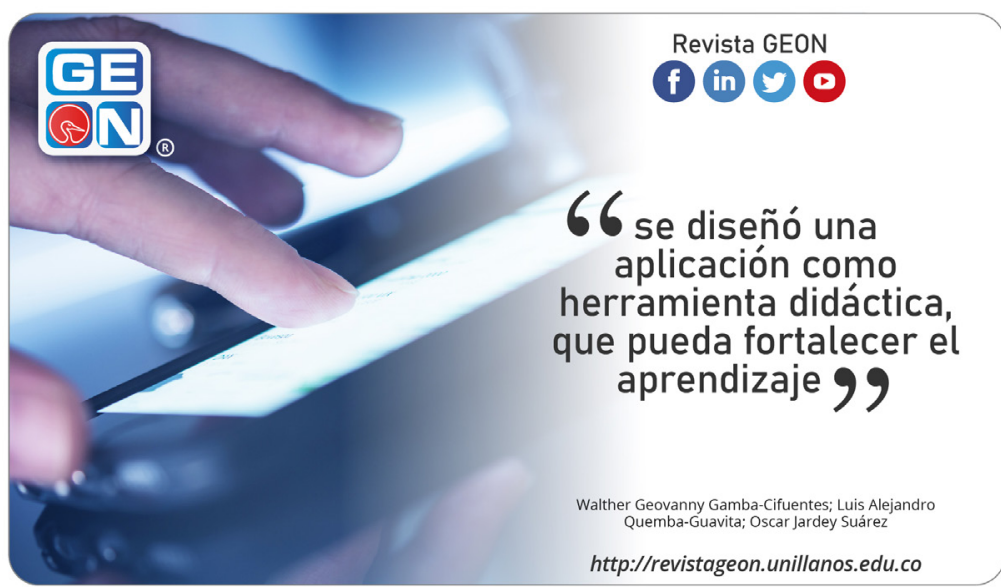

desarrollada con el programa Xcode ${ }^{\circledR}$ y lenguaje de programación SwiftUI ${ }^{\circledR}$. Los resultados indican que apoyarse en la metodología ISD-MeLO resulta pertinente; sin embargo, elegir un modelo pedagógico que soporte el diseño y desarrollo de la aplicación da mayor coherencia. A manera de conclusión, una forma innovadora de construir recursos educativos digitales como herramienta para el aprendizaje está en articular adecuadamente las posibilidades de la ingeniería de software con la propuesta educativa que subyace, en este caso, la aplicación Physics Pocket.

Palabras clave: ambiente de la clase, educación básica, enseñanza de la física, recursos educativos abiertos, relación profesor-alumno, tecnología educacional.

Códigos JEL: 120 Generalidades, O32 Gestión de la innovación tecnológica

\section{Abstract}

Cómo citar este artículo / Toreference this article:

Gamba Cifuentes, W. G., Quemba Guavita, L. A., \& Suárez, O. J. (2021). Diseño y desarrollo de una aplicación como herramienta innovadora para el aprendizaje de la ley de los gases ideales. Revista GEON (Gestión, Organizaciones Y Negocios), 8(1), e-270. https://doi. org/10.22579/23463910.270
The objective of this work is to report the design and development of an App in the iOS system for the study of the ideal gas law with $11^{\text {th }}$ grade students at a private school in Bogotá, Colombia, since in recent years this topic has generated low levels. Therefore, an App has been designed as a teaching tool that can strengthen learning. Different sources of work point out that the use of Information and 
Cómo citar este artículo / Toreference this article:

Gamba Cifuentes, W. G., Quemba Guavita, L. A., \& Suárez, O. J. (2021). Diseño y desarrollo de una aplicación como herramienta innovadora para el aprendizaje de la ley de los gases ideales. Revista GEON (Gestión, Organizaciones Y Negocios), 8(1), e-270. https://doi. org/10.22579/23463910.270
Communication Technologies, such as APPs, can potentially favor learning. The methodology followed for the design and development is ISD-MeLO, which focuses on the outline and progress of educational content and is based on the ADDIE instructional design model; the phases are: 1) Analysis, 2) Design, 3) Development, 4) Implementation and 5) Evaluation. The App was developed with the $X \operatorname{code}^{\circledR}$ program and SwiftUI ${ }^{\circledR}$ programming language. The results indicate that relying on the ISD-MeLO methodology is pertinent, however choosing a pedagogical model that supports the design and development of the App gives greater coherence. In conclusion, an innovative way to build digital educational resources, as a tool for learning, is to adequately articulate the possibilities of software engineering with the educational proposal underlying the "Physics Pocket" App in this case.

Keywords: Basic education; Classroom environment; Educational technology; Open educational resources; Science teaching; Teacher-student relationship.

\section{Introducción}

Physics Pocket App es el desarrollo de una aplicación como recurso educativo digital (RED), utilizando la herramienta de desarrollo Xcode ${ }^{\circledR} 11$ y el lenguaje SwiftUl ${ }^{\circledR}$. Esta aplicación establece la enseñanza de la ley de gases ideales (Young \& Feedman, 2009) para estudiantes de undécimo grado, de una escuela privada de la ciudad de Bogotá. La aplicación se basa, desde lo pedagógico, en el emergente modelo conectivista (Siemens, 2004), las comunidades de aprendizaje (Gairín, 2006), el uso de las tecnologías de la información y la comunicación (TIC) y el aprendizaje en la era digital (Sierra, 2010); cada uno de estos elementos busca consignarse en la aplicación. Algunos estudios iniciales reportan un uso intenso, del 78\% para los estudiantes y del 70\% para los docentes, en espacios dedicados a la educación y comunicación a través de los dispositivos móviles (Basantes, Naranjo, Gallegos \& Benítez, 2017). El estudiante asume el papel principal en su proceso de formación, mientras que el docente estará orientando y acompañando el proceso de aprendizaje apoyado en dispositivos móviles, en general las TIC.

\section{Contexto teórico}

Desde el punto de vista disciplinar, se eligió el tema de gases ideales para tra- 
bajar con los alumnos de undécimo grado, debido a que es un tema que se aborda en el curso, en la sección de termodinámica. Además, en ausencia de un laboratorio formal en la institución, el desarrollo de actividades a través de simulaciones por computadora permite, de manera interactiva, estudiar el comportamiento y las características de un sistema termodinámico. Esta aplicación se encuentra enfocada en el marco de la enseñanza de la física orientado por las ciencias naturales, en el colegio San Juan de Ávila. En esta institución, se evidencia una pedagogía del acompañamiento y el buen uso de los dispositivos móviles. A partir de esto, se busca que los estudiantes desarrollen el pensamiento científico mediante situaciones problema y el aprendizaje activo partiendo de un análisis completo de los fenómenos naturales en los cuales pueda verse inmerso. No obstante, la institución carece de un laboratorio o instalaciones en las cuales el aprendizaje mediante la experimentación pueda llevarse a cabo, por lo cual el uso de herramientas digitales y tecnológicas es una opción continua para docentes y estudiantes de la institución, que aporta a los procesos de enseñanza aprendizaje (Morales, Moreno, Romano \& García, 2020). Dicho esto, se establece el desarrollo de la aplicación, como un RED, para favorecer el estudio de la física, en particular de los gases ideales.

\section{Materiales y métodos}

Para el diseño y desarrollo se emplea la metodología ISD-MeLO, sigla que proviene de Instructional Systems Development Methodology based on e-Learning Objects (Baruque \& Melo, 2014). El modelo ISD-MeLO está fundamentado en los parámetros instruccionales de ADDIE (Analysis, Design, Development, Implementation and Evaluation). Bajo la óptica de este modelo, se trabajan las etapas necesarias para producir la aplicación; estas son: análisis, diseño, desarrollo, implementación y evaluación (ADDIE), tal como se observa en la figura 1.

Figura 1. Fases del modelo ADDIE. Fuente: propia de los autores.

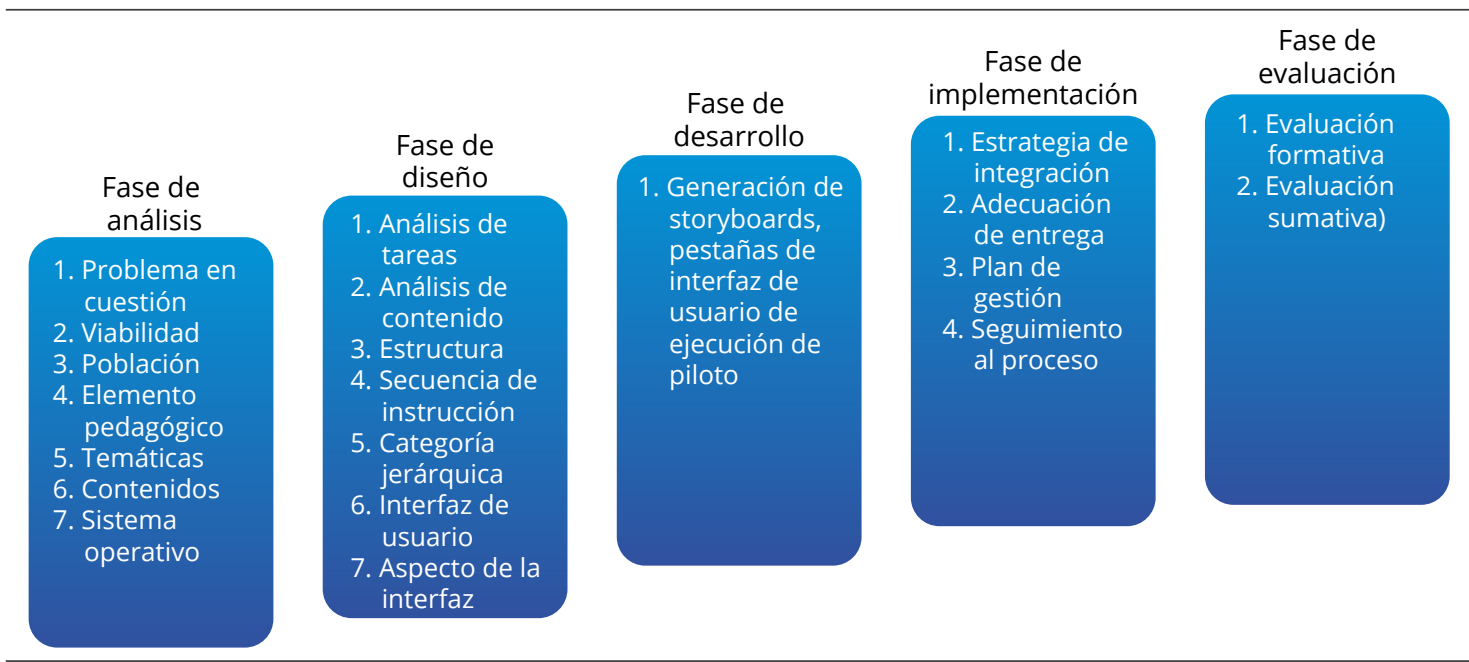


El software en el que se desarrolló la aplicación es $X \operatorname{code}^{\circledR}$, la que se constituye por defecto como el editor de diseño del programador de Apple ${ }^{\circledR}$. Por su parte, SwiftUI ${ }^{\circledR}$ es la pieza de software para crear interfaces de usuario acopladas a Xcode ${ }^{\circledR}$ en los diferentes sistemas operativos de Apple ${ }^{\circledR}$; este software se acopla perfectamente con $\mathrm{Xcode}^{\circledR}$, de tal manera que las interfaces de usuario se ajustan a los diferentes dispositivos de Apple ${ }^{\circledR}$ utilizando el conjunto de herramientas y API (Application Programming Interface).

\section{Resultados}

A partir de las fases del modelo ADDIE, se regulan las funcionalidades que debe tener la aplicación que será construida. Como primera medida, se establece la exploración de la herramienta de diseño $X \operatorname{code}^{\circledR}$ y el lenguaje de programación SwiftUI ${ }^{\circledR}$. Esto para hacer una revisión de librerías, comandos, compatibilidad y operabilidad con el desarrollo del RED. Se establece de igual forma una interfaz de rapidez y fluidez en el diseño instruccional, que permite una ejecución óptima en el manejo de herramientas tecnológicas por parte de los autores. De acuerdo con la revisión de herramientas y creación de aplicaciones y lenguaje de programación, se establece la viabilidad en la creación de la aplicación. El desarrollo del diagrama de análisis comprende los ejes centrales y transversales bajo los cuales está soportado el RED. En este caso, se establecen como ejes centrales: las herramientas de desarrollo del RED, el elemento pedagógico y la enseñanza de la física. Mientras que, como ejes transversales, se establecen las herramientas de diseño y programación, el estilo y medio de aprendizaje y los componentes conceptuales de contenido.

X code $^{\circledR}$ y Swiftul ${ }^{\circledR}$ son las piezas de software que posibilitan la generación del contenido informático y tecnológico. La compilación de la aplicación se realiza en tiempo real, en tanto se va programando; es decir, la interfaz de desarrollo permite la visualización permanente de cómo va quedando la aplicación (figura 2).

Figura 2. Diagrama de análisis. Fuente: propia de los autores.

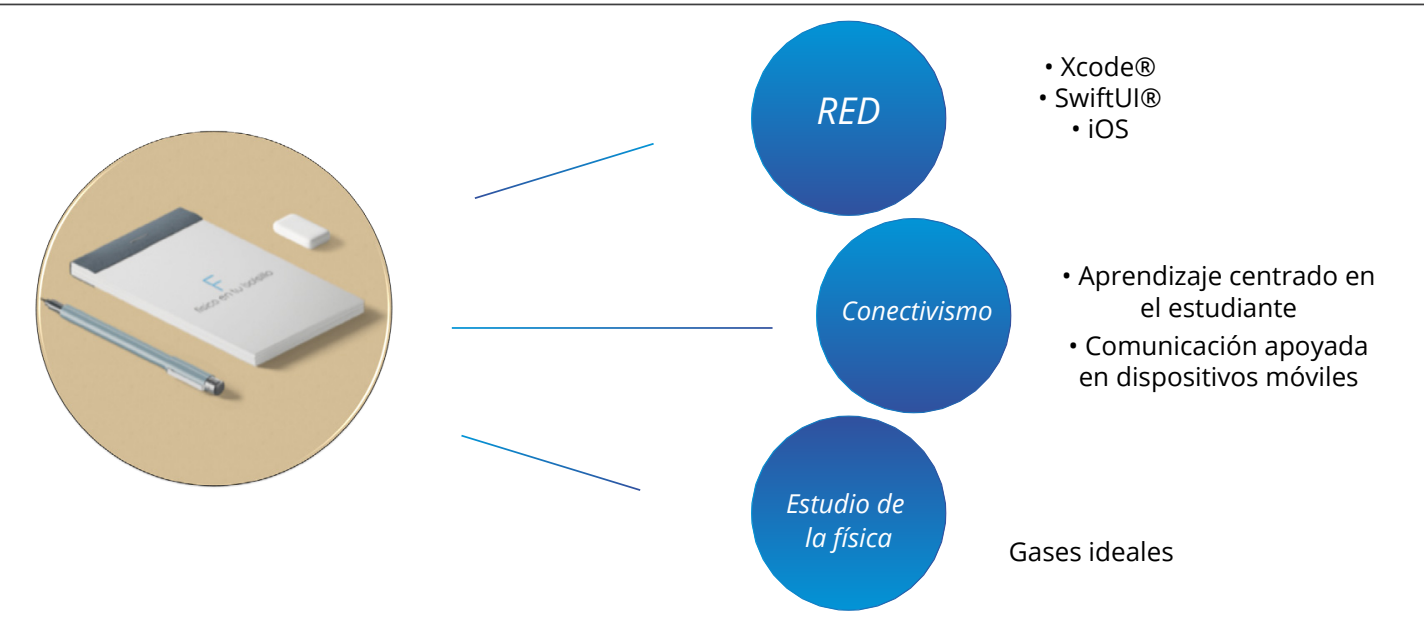


Para el desarrollo de la aplicación, se utilizó la versión de $X_{\text {code }}{ }^{\circledR}$ 11.4.1 (11E503a), bajo el estilo declarativo proporcionados por SwiftUl ${ }^{\circledR}$ para algunos elementos animados. Para la animación en tiempo de ejecución, se controló el sistema para garantizar los movimientos programados, así como la estabilidad de la aplicación.

Como se observa en la figura 3, asumiendo al diseño y desarrollo como etapas intensivas e interactuantes, se atiende la siguiente ruta: (a) generación del storyboard (mediante Launch Screen); (b) generación del home; (c) contenido; (d) implementación del contenido conceptual mediante NavigationView ${ }^{\circledR}$ y List $^{\circledR}$ y visualización de la información mediante Navigation-
Link $^{\circledR}$; (e) implementación de aplicaciones mediante List ${ }^{\circledR}$, NavigationLink ${ }^{\circledR}$ y NavigationView ${ }^{\circledR}$; (f) incrustamiento del canal de YouTube ${ }^{\circledR}$ mediante WebView $^{\circledR} ;$ (g) incrustamiento de la página $\mathrm{PHET}^{\circledR}$ de la Universidad de Colorado, mediante WebView ${ }^{\circledR}$; (h) unidad de soporte y acceso a la página web de Physics Pocket App ${ }^{\circledR}$.

Finalmente, la aplicación diseñada se fundamenta en un entorno "minimalista". Los marcos, las imágenes y los bordes son el punto de referencia para el diseño del ícono de la aplicación al momento de incluirla en la Appstore ${ }^{\circledR}$ y de igual manera establece los parámetros de diseño de la página web de la aplicación, como se muestra en la figura 4.

Figura 3. Modelo ADDIE: desarrollo. Fuente: propia de los autores.

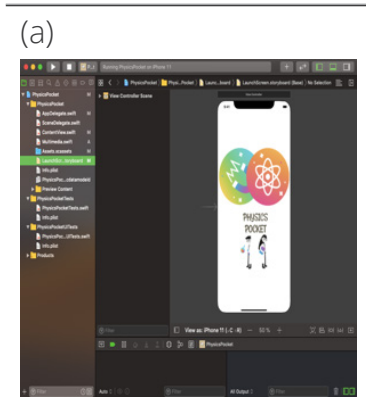

Storyboard

(e)

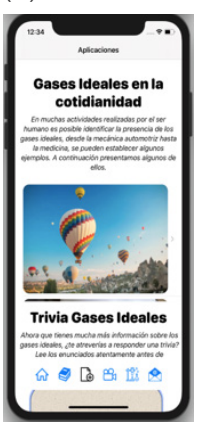

Aplicaciones (b)

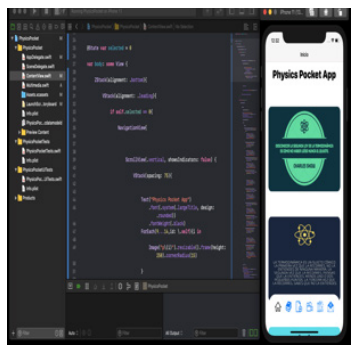

Home

$(f)$

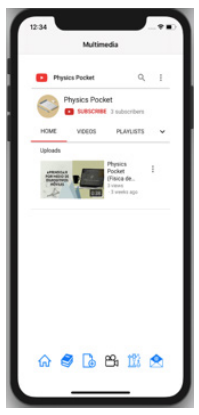

Canal de YouTube
(C)

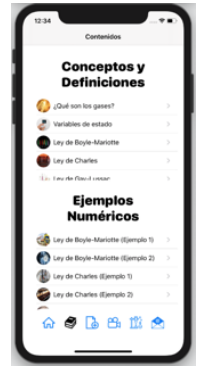

Contenido

(g)

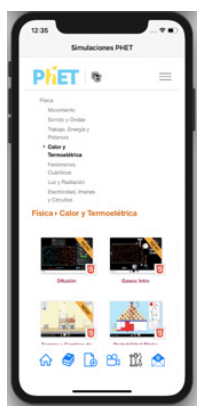

Simulaciones PHET (d)

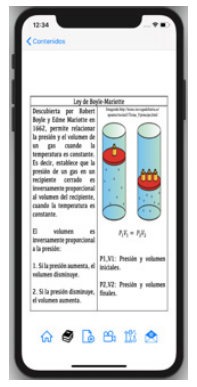

Contenido conceptual

(h)

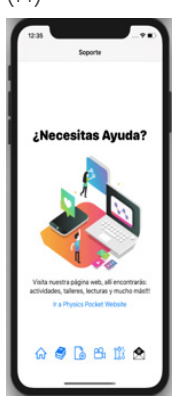

Soporte 
Figura 4. Diseño página web. Fuente: propia de los autores.

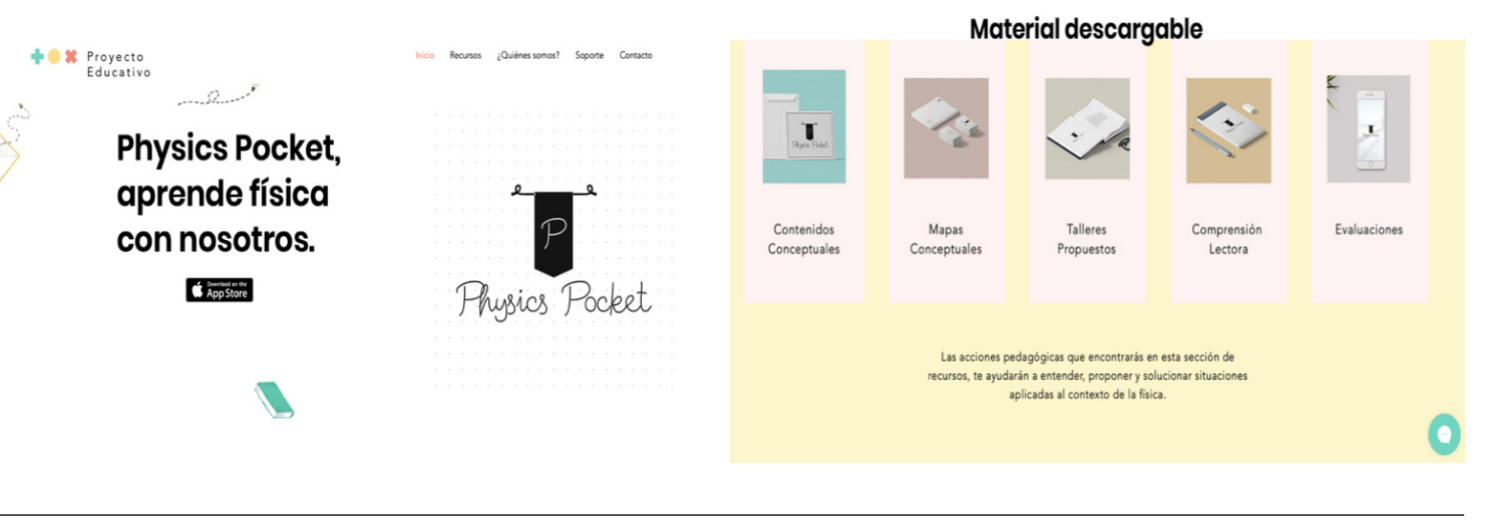

\section{Discusión}

La metodología ISD-MeLO posibilita estructurar el diseño y la diagramación de aplicaciones móviles a partir de un contexto educativo específico. Dado que ISD-MeLO se fundamenta en el modelo instruccional ADDIE; el diseño y desarrollo, en conjunción con el conectivismo; es posible llevarlo a que esté centrado en el estudiante, lo que potencialmente puede ser incorporado en rutas de aprendizaje que promuevan aprendizajes autónomos.

El diseño de la aplicación coincide con la mirada de Suárez y Ortegón (2011), en la medida en que se pone en evidencia la importancia de conocer las necesidades de los estudiantes. Para estos autores, que proponen dos conceptos clave de la física como parte de la formación académica de los ingenieros, el centro de masa y el centro de gravedad, resulta plausible poner en el centro de la discusión el aprendizaje de los estudiantes, toda vez que es por ellos que finalmente se orienta el acto educativo (Suárez \& Ortegón, 2011). A pesar de que estos autores trabajan temas de física para ingenieros a través de una aplicación orientada al favorecimiento del pensamiento matemático, se logra visionar a la aplicación como una herramienta para la conceptualización del centro de masa. (Suárez \& Ortegón, 2011). Para el caso que expone este trabajo, la esta aplicación se concibe como una mediación en pro del aprendizaje de los gases ideales, que va más allá.

Sandoval y Carvajal (2016) expresan la utilidad de un diseño metodológico y exploratorio a partir del uso de las TIC, como estrategia de reconocimiento. Si bien la aplicación desarrollada por estos autores se fundamenta en "entrada, proceso y salida", no incluye explícitamente, en la etapa de diseño y desarrollo, una orientación pedagógica que base las decisiones que se toman en esta etapa (Sandoval \& Carvajal, 2016). Lo anterior es diferente al trabajo que aquí se expone y que de alguna forma da una mayor pertinen- 
cia a la generación de aplicaciones orientadas a la educación (Salamanca, Uribe \& Mendoza Vega, 2017).

Considerar la visualización en el diseño y desarrollo resulta una condición necesaria (Sandoval \& Carvajal, 2016; Suárez \& Ortegón, 2011). LOS RED requieren de una interfaz de visualización pertinente y adecuada a la población estudiantil objeto de estudio, lo que implica que considerar el diseño minimalista resulta una decisión importante en la generación de las aplicaciones orientadas a la educación.

El sistema operativo, la población a la cual la aplicación está dirigida y las TIC, todos establecidos como punto de partida para la creación de la aplicación, le dan un propósito ajustado con total pertinencia. Delimitar el problema a una población específica permite conocer en mayor profundidad la necesidad que debe resolverse y a su vez establecer de qué manera una aplicación puede dar solución total a este. En este trabajo se señala con claridad la necesidad de apoyarse en una tendencia pedagógica-didáctica (conectivismo), que posibilite el tratamiento del tema objeto de estudio (gases ideales). Lo anterior demanda del equipo de diseño un hilo conductor que, en forma creativa, el equipo autor de las aplicaciones debe consolidar; en ese ejercicio, la metodología ISD-MeLO resulta adecuada.

Finalmente, bajo la mirada de la enseñanza de la física en la era digital y la implementación de ambientes virtuales de aprendizaje, objetos virtuales de aprendizaje, recursos edu- cativos abiertos y modelo pedagógico conectivista, el fundamento teórico consultado establece un paralelo y/o similitud considerable con los trabajos de investigación que han implementado estrategias en el aula de clase bajo las categorías mencionadas anteriormente.

\section{Conclusiones}

La metodología ISD-MeLO, en sus fases de análisis, diseño, desarroIlo, implementación y evaluación, se constituye en una forma adecuada de producir una aplicación para la educación, siempre y cuando se consideren el contexto educativo de la población estudiantil destino, debidamente acotado el contenido disciplinar y el fundamento pedagógico.

Se permite señalar que una aplicación orientada al ámbito educativo necesita estar localizada disciplinaria y pedagógicamente en la población estudiantil que se beneficia. Determinar los gases ideales, como tema disciplinar, permitió focalizar el desarrollo e incorporación de recursos adecuados; así mismo, elegir el conectivismo como sustento pedagógico permite determinar las características propias de cada una de las actividades que se implementaron.

La aplicación Physics Pocket App ${ }^{\circledR}$ se constituye en un recurso educativo digital innovador, que emerge de un diseño propio de la ingeniería, articulado debidamente con el entono educativo, hecho que, potencialmente, puede indicar que es un material educativo pertinente para jóvenes que están en la educación media. 


\section{Referencias}

Baruque, L. B. \& Melo, R. N. (2004). Learning theory and instruction design using learning objects. Journal of Educational Multimedia and Hypermedia, 13(4), 343-370. https://bit.ly/3r1STIN

Basantes, A., Naranjo, M., Gallegos, M. \& Benítez, N. (2017). Los dispositivos móviles en el proceso de aprendizaje de la facultad de educación ciencia y tecnología de la universidad técnica del norte de Ecuador. Formacion Universitaria, 10(2), 79-88. https://bit. ly/3szihpu

Gairín-Sallán, J. (2006). Las comunidades virtuales de aprendizaje. Educar, 37, 41. https://bit.ly/3bOGjpl

Morales, M. G., Moreno, K. C., Romano, M. M. del S. \& García, M. del R. (2020). Gestión del conocimiento, a través de plataformas y herramientas digitales de aprendizaje ante la migración de clases presenciales a en linea. Revista Gestión, Organizaciones y Negocios, 7(2), 1-19. https://bit.ly/2NDfNI8

Salamanca, E. J., Uribe, C. A. \& Mendoza, L. M. (2017). ¿Cómo desarrollar un nuevo mercado donde la competencia no tiene ninguna? Dictamen Libre, (20), 107-120. https://bit.ly/2Mv1UuO
Sandoval, C. A. \& Carvajal-Beltrán, L. V. (2016). Aplicación móvil de realidad aumentada para la ubicación de las aulas de clase en el Campus Porvenir de la Universidad de la Amazonia. Revista Científica, (26), 84-94- https://bit. ly/3dTYSLY

Siemens, G. (2004). Conectivismo: una teoría del aprendizaje para la era. Publicado bajo Licencia Creative Commons 2.5. Recuperado el 2019, de https://www.comenius.cl/recursos/virtual/minsal_v2/Modulo_1/Recursos/ Lectura/conectivismo_Siemens.pdf

Sierra, I. (2010). Estrategias de mediación metacognitiva en ambientes convencionales y virtuales: influencia en los procesos de autorregulación y aprendizaje autónomo en estudiantes universitarios [tesis de doctorado, Universidad de Granada]. Repositorio de la Universidad de Granada. https://bit.ly/3q2hdZw

Suárez, O. J. V., \& Ortegón, I. Y. C. (2011). Matematización del centro de masa a partir de una aplicación en la ingeniería. Revista científica, 1(13), 36-39. https://doi.org/10.14483/23448350.618

Young, H. \& Freedman, R. (2009). Física universitaria. Pearson Educación. 